MATEC Web of Conferences 22,02007 (2015)

DOI: $10.1051 /$ matec conf/ 20152202007

(c) Owned by the authors, published by EDP Sciences, 2015

\title{
A Study on the Data Compression Algorithm of Power Quality Based on Wavelet Transformation
}

\author{
Wenxiong Peng * \\ The State Key Laboratory of Power Transmission and Distribution Equitment \& System Security and New techno \\ logy, College of Electric Engineering, Chongqing University, Chongqing, China \\ Jun Yuan \\ Jiangxi Power Co., Ltd of State Grid in Xingan, Ji'an, Jiangxi, China \\ Zhihong Fu \\ College of Electric Engineering, Chongqing University, Chongqing, China
}

\begin{abstract}
Based on the development history of wavelet transformation's applications in data compression, this paper studies thee wavelets' property of being continuous and discrete, establishes a power quality data compression model of wavelet transformation, discusses the threshold coefficient compression algorithm after the wavelet transformation, and makes an improvement with the low-frequency, high-frequency and self-adaptive power quality of the threshold compression algorithm. In the end, this paper verifies through a simulation experiment that the algorithm is adaptable to the compression of power quality data and signals are able to maintain a relatively low distortion.
\end{abstract}

Keywords: wavelet transformation; power quality; data compression algorithm

\section{INTRODUCTION}

With the rapid development of economy, the modern power load structure changes tremendously and the power quality conflict becomes increasingly prominent. The increase of electricity demand and the increase of user's demand for electricity, especially the improvement of sophisticated electronics such as computer, lead to the growing demand for power quality. However, the power quality has not been improved. The start of such large non-linear electrical equipment as the electric arc furnace would cause an impact effect on the power grid, decrease the power quality and reduce the quality of products to some extent. Meanwhile, the insecurity of electronic equipment brings about great difficulties to the production life.

In recent years, many scholars have carried out studies on this in order to improve the power quality. For example, in 2014, Peixia Sun et al. ${ }^{[1]}$ carried out a study on problems of the power quality data compression, established a model combining the high-frequency compression and the lossless compression, and conducted a high-frequency compression with the improved triple matrix. The final result indicated that the algorithm has a good compression ratio. In 2013, Xiao Tian ${ }^{[2]}$ conducted a study on the method of power quality signal compression of wavelet transformation, established an intervening model in accordance with power quality signal problems, and carried out a system simulation in line with power quality signals. The result proved that the compression algorithm is able to obtain the original signals with smaller errors and the high probability. In 2010, Lan $\mathrm{Li}^{[3]}$ carried out an intensive study on such problems as ever-increasing data flow and decreasing network speed from the angle of wavelet transformation and cluster compression, classifying the data with numbers, compressing the data through the method of cluster compression and verifying through simulations in line with the principle of data similarity. The result indicated that the compression precision of power data can be improved by the clustering with Euclidean distance.

Based on previous studies, this paper carries out a further analysis and research on the power quality data compression algorithm of wavelet transformation, establishes the coefficient compression algorithm of the two-dimensional wavelet transformation, realizes the transfer of signal power and the compression of wavelet low-frequency data successfully, and conducts a simulation test. The result indicates that the method has a fast speed and a good compression effect.

\section{THEORETICAL MODELS OF THE DATA COMPRESSION ALGORITHM OF WAVELET TRANSFORMATION}

In1980s, wavelet transformation grew rapidly as a new discipline in this field with the rapid development

*Corresponding author: $\underline{\mathrm{dx} 761213 @ 163 . c o m}$

This is an Open Access article distributed under the terms of the Creative Commons Attribution License 4.0, which permits unrestricted use, distribution, and reproduction in any medium, provided the original work is properly cited. 
of applied mathematics. At present, it has become a hot topic of the society. Being similar to the Fourier transformation, the compression algorithm of wavelet transformation also decomposes signals. But the difference is that the compression algorithm decomposes signals after the superposition. Thus, it is known as a "mathematical microscope".

\subsection{Wavelet basis function model}

Wavelets with such properties as positive/negative alternation and volatility usually refer to waves with small areas within a limited range.

$\psi_{a, b}(\mathrm{t})=\mid a^{\frac{1}{2}} \psi\left(\frac{t-b}{a}\right) \quad \mathrm{a}, \mathrm{b} \in \mathrm{R}, \mathrm{a} \neq 0$

The formula mentioned earlier is a wavelet generating function, in which the horizontal movement scale is $\mathbf{a}$ and the vertical movement scale is b. In order to realize the Fourier transformation, $\psi_{a, b}(\mathrm{t})$ should satisfy as follows:

$c_{\psi}=\int_{R} \frac{|\psi(\omega)|^{2}}{\omega} d \omega<\infty$

The formula mentioned earlier proves that the volatility of wavelets and the mean value is 0 .

The function formed by different levels of horizontal and vertical movement of wavelets is called the wavelet basis function.

\subsection{Wavelet model of haar}

The wavelet with the longest time and most properties is called haar wavelet. Select a coefficient set of two scales in the case of Formula (2) is satisfied, and there is only one coefficient as follows:

$h(\mathrm{n})=\left\{\frac{1}{\sqrt{2}}, \frac{1}{\sqrt{2}}\right\}$

The normalized scaling function can be expressed through the above formula as follows:

$\varphi(t)= \begin{cases}1 & \text { if } 0<t<1 \\ 0 & \text { other }\end{cases}$

The wavelet function is shown as follows:

$\psi(t)= \begin{cases}1 & \text { if } 0<t<1 \\ -1 & \text { if } \frac{1}{2}<t<1 \\ 0 \quad \text { other }\end{cases}$

It can be summarized from the above process that $L^{2}(\mathrm{R})$ formed by the horizontal and vertical movement is a set of normalized orthogonal basis.
2.3 Continuous wavelet transformation model

If $\psi(t)$ is the mother wavelet and $\psi_{a, b}(t)=|a|-\frac{1}{2} \psi\left(\frac{t-b}{a}\right), \quad a \in R-\{0\}, b \in R \quad, \quad$ the signal is $f(\mathrm{t}) \in L^{2}(\mathrm{R})$, the continuous wavelet transformation about $f(\mathrm{t})$ can be expressed as follows:

$$
\begin{aligned}
& W f(\mathrm{a}, \mathrm{b})=C W T(\mathrm{a}, \mathrm{b})=\left\langle\mathrm{f}(\mathrm{t}), \psi_{a, b}(t)\right\rangle \\
& =\int f(\mathrm{t})|a|^{-\frac{1}{2}} \bar{\psi}\left(\frac{t-b}{a}\right) d t
\end{aligned}
$$

Where, the real-valued function is $\psi(t)$. The above formula can be further optimized as follows:

$$
\begin{aligned}
& W f(\mathrm{a}, \mathrm{b})=C W T(\mathrm{a}, \mathrm{b})=\left\langle\mathrm{f}(\mathrm{t}), \psi_{a, b}(t)\right\rangle \\
& =\int f(\mathrm{t})|a|^{-\frac{1}{2}} \psi\left(\frac{t-b}{a}\right) d t
\end{aligned}
$$

With the combination of the above formula, the reconstruction formula of wavelet transformation can be expressed as follows:

$$
\mathrm{f}(\mathrm{t})=\frac{1}{c_{\psi}} \int_{-\infty}^{+\infty} \int_{-\infty}^{+\infty} W f(\mathrm{a}, \mathrm{b})\left[|a|^{-\frac{1}{2}} \psi\left(\frac{t-b}{a}\right)\right] d b \frac{d a}{a^{2}}(8)
$$

Where, $c_{\psi}=\int_{0}^{+\infty} \frac{|\hat{\psi}(\omega)|^{2}}{\omega} d \psi$

The above-mentioned one-dimensional wavelet is further improved. Assume that $\psi(\mathrm{x}, \mathrm{y})$ is a two-dimensional wavelet function, the signal function is expressed as $\mathrm{f}(\mathrm{x}, \mathrm{y}) \in \mathrm{L}^{2}\left(\mathrm{R}^{2}\right) \quad, \quad$ and $\psi_{a, b, \mathrm{c}}(\mathrm{x}, \mathrm{y})=|a|^{-1} \psi\left(\frac{x-b}{a}, \frac{y-c}{a}\right)$, so it is shown as follows:

$$
2-C W T(\mathrm{a}, \mathrm{b}, \mathrm{c})=\iint f(\mathrm{x}, \mathrm{y}) \psi_{a, b, \mathrm{c}}(\mathrm{x}, \mathrm{y}) \mathrm{dxdy}
$$

The above formula is the transformation function of the two-dimensional wavelet. If the dual function of the generating function is expressed as $|\hat{\psi}(\mathrm{x}, \mathrm{y})|=|a|^{-1} \hat{\psi}\left(\frac{x-b}{a}, \frac{y-c}{a}\right)$, then the reconstruction formula of wavelet is shown as follows: $f(\mathrm{x}, \mathrm{y})=\iint f(\mathrm{x}, \mathrm{y}) \hat{\psi}_{a, b, \mathrm{c}}(\mathrm{x}, \mathrm{y}) \mathrm{dxdy} \psi_{a, b, \mathrm{c}}(\mathrm{x}, \mathrm{y})$ 


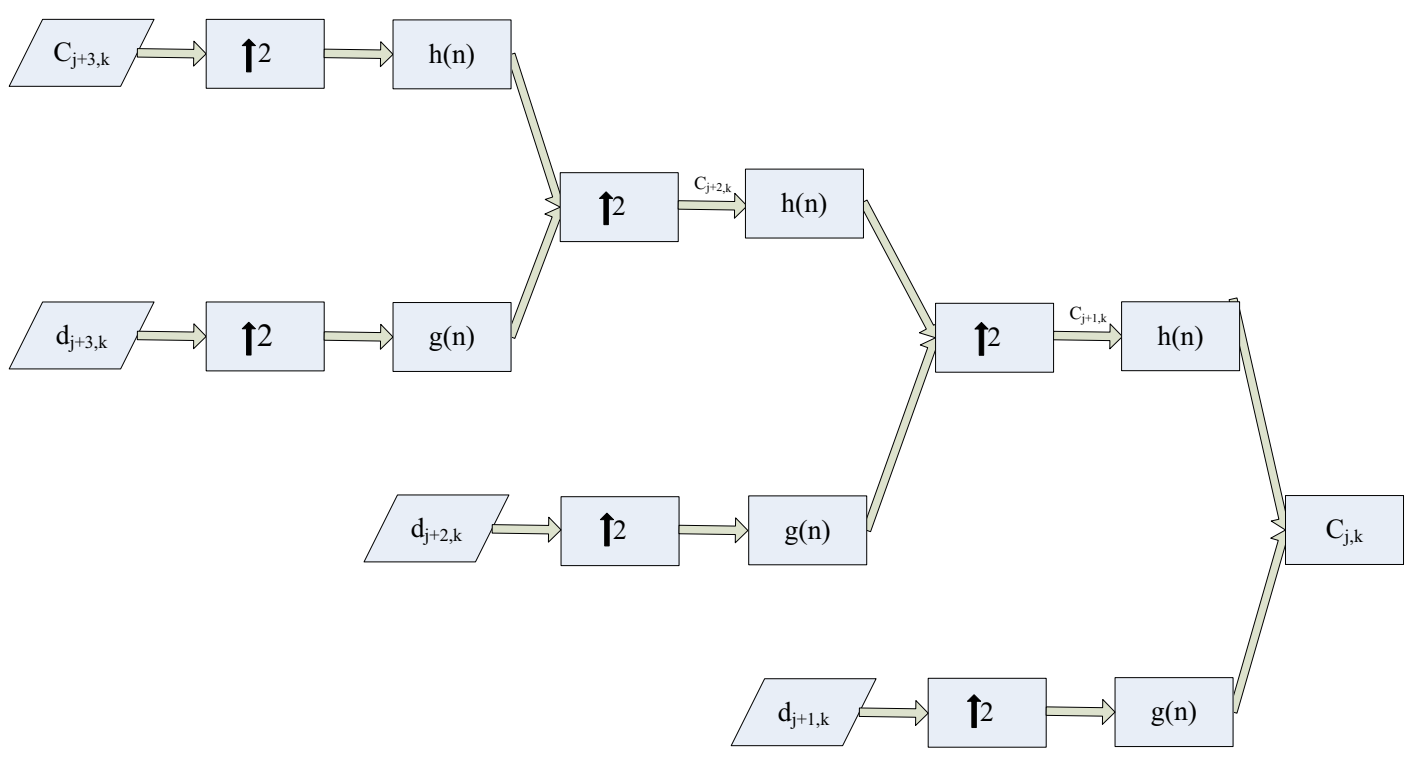

Figure 1. Diagram of signal reconstruction process diagram

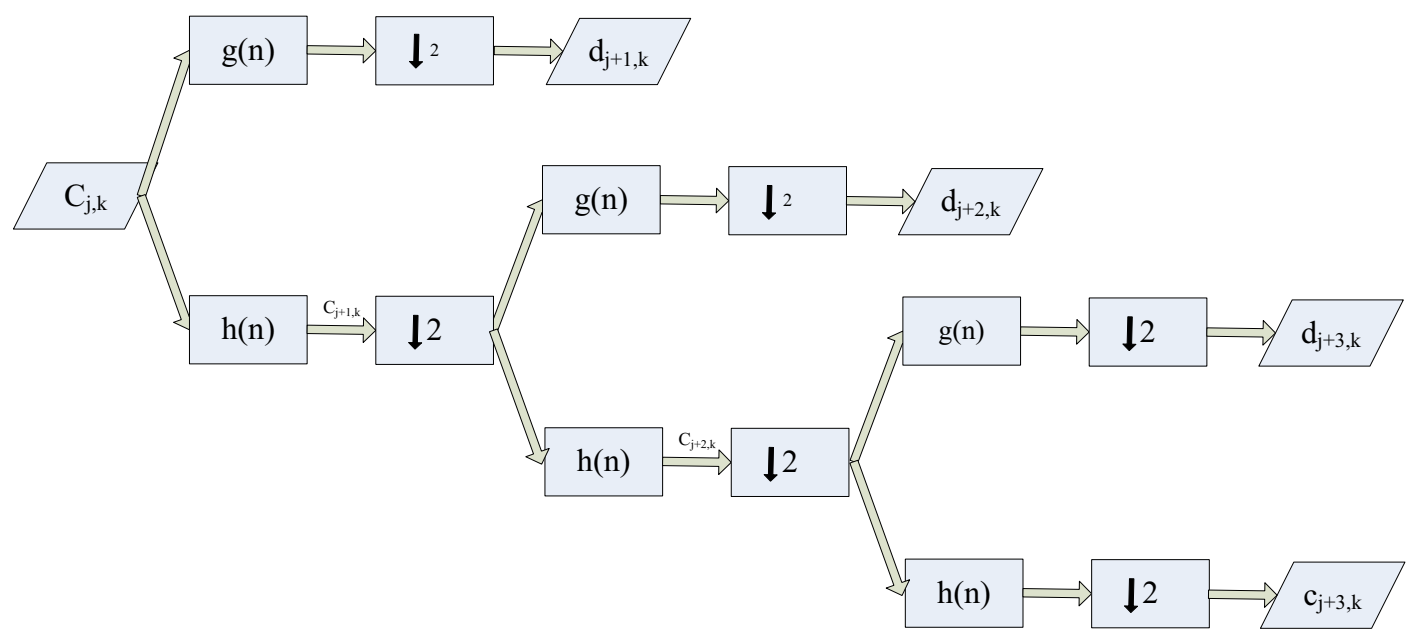

Figure 2. Diagram of multi-dimension wavelet decomposition process diagram

\subsection{Discrete wavelet transformation}

The continuous wavelet transformation increases the difficulty of the wavelet transformation structure. Therefore, it is replaced by discrete wavelet transformation so as to improve the effects of interpretation and analysis.

In the above-mentioned continuous wavelet transformation, if coefficients $\mathrm{a}, \mathrm{b}$ of $\psi_{a, b}(\mathrm{x})$ are quan- tized in line with the binary system or multiples of the binary system, then $\mathrm{a}=2^{-m}, \mathrm{~b}=\mathrm{n} 2^{-m}, m, n \in Z$ and $\psi_{\mathrm{m}, \mathrm{n}}(\mathrm{k})=2^{\frac{m}{2}} \psi\left(2^{m} \mathrm{k}-\mathrm{n}\right)$. Thus, the corresponding discrete wavelet can be expressed as follows:

$\operatorname{DWT}(\mathrm{m}, \mathrm{n})=2^{\frac{m}{2}} \sum_{k} f(\mathrm{k}) \psi\left(2^{m} \mathrm{k}-\mathrm{n}\right)$ 
The reconstruction formula which is relevant to the above formula is shown as follows:

$$
f(\mathrm{k})=\sum_{m} \sum_{n} \operatorname{DWT}(\mathrm{m}, \mathrm{n}) \psi_{\mathrm{m}, \mathrm{n}}(\mathrm{k})
$$

The variable of the two-dimensional discrete wavelet is discretized and expressed as follows:

$$
2-\mathrm{DWT}\left(\mathrm{J}, \mathrm{K}_{1}, \mathrm{~K}_{2}\right)=\sum_{m} \sum_{n} f(\mathrm{~m}, \mathrm{n}) \psi_{\mathrm{j}, \mathrm{k}_{1}, \mathrm{k}_{2}}(\mathrm{~m}, \mathrm{n})(13)
$$

The reconstruction formula function of the continuous transformation of the two-dimensional wavelet is then discretized to obtain the formula as follows:

$$
\mathrm{f}(\mathrm{m}, \mathrm{n})=\left\langle\mathrm{f}(\mathrm{m}, \mathrm{n}), \hat{\psi}_{\mathrm{j}, \mathrm{k}_{1}, \mathrm{k}_{2}}(\mathrm{~m}, \mathrm{n})\right\rangle \psi_{\mathrm{j}, \mathrm{k}_{1}, \mathrm{k}_{2}}(\mathrm{~m}, \mathrm{n})
$$

\subsection{Discrete wavelet transformation Mallat model}

In order to enhance the degree of discrimination and analysis, scholars of the signal analysis proposed the Mallat model in 1980s. The specific algorithm is provided as follows:

$$
\begin{aligned}
& c_{j, k}=\sum_{m} h(\mathrm{~m}-2 \mathrm{k}) \mathrm{c}_{j-1, m} \\
& d_{j, k}=\sum_{m} h(\mathrm{~m}-2 \mathrm{k}) \mathrm{g}_{j-1, m}
\end{aligned}
$$

Where, coefficients@ represent the highest and the lowest points of the decomposition of the filter. The rapid reconstruction algorithm of the discrete wavelet filter is expressed as follows:

$$
c_{j-1, m}=\sum_{k} c_{j, k} \overline{h(\mathrm{~m}-2 \mathrm{k})}+\sum_{k} d_{j, k} \overline{\mathrm{g}(\mathrm{m}-2 \mathrm{k})}
$$

In the above formula, the highest and the lowest points of the reconstruction end of the filter are respectively $\bar{g}, \bar{h}$. The wavelet coefficient and the scale coefficient are respectively ${ }^{c} j, k$ and $d_{j, k}$.

Structures of the wavelet reconstruction and the decomposition algorithm are shown in Figure 1 and Figure 2.

Different wavelets usually have different time-domain properties. In the above figure, various results are obtained from the processing of one signal. The selection and optimization of a wavelet are generally adopted in the process of power quality data compression.

\subsection{Data compression model of wavelet transfor- mation}

\subsubsection{Threshold compression algorithm of} low-frequency coefficient

Coefficients should be further processed after the wavelet transformation due to the lack of the compression function. The non-uniform distribution of the original data is the result obtained from the wavelet transformation. It is able to realize the acceptance or rejection of coefficients of the high-frequency part as well as the retention of low frequency coefficients so as to achieve the objective of compression.

The compression of wavelet threshold is a key step in the compression algorithm of wavelet transformation. Steps of compressing signals are shown as follows:

1) Select suitable decomposing layers from the signal $f(\mathrm{t})$ after the transformation so as to obtain the corresponding coefficient $\omega_{j, k}$.

2) Use thresholds to process in order to estimate the wavelet coefficient $\hat{\omega}_{j, k}$.

Soft threshold:

$\hat{\omega}_{j, k}=\left\{\begin{array}{r}\operatorname{sign}\left(\left|\omega_{j, k}\right|-\lambda\right),\left|\omega_{j, k}\right| \geq \lambda \\ 0,\left|\omega_{j, k}\right|<\lambda\end{array}\right.$

Hard threshold:

$\hat{\omega}_{j, k}=\left\{\begin{array}{r}\omega_{j, k},\left|\omega_{j, k}\right| \geq \lambda \\ 0,\left|\omega_{j, k}\right|<\lambda\end{array}\right.$

Where, the standard deviation and the length of the signal are respectively $\delta$ and $N . \lambda=\sqrt{2 \log N \delta}$.

Systematic statistics of the data in the source data flow are provided in order to further improve the data compression algorithm of wavelet transformation. Set a reasonable threshold $m$ in line with the results and numbers that are larger than the threshold which is taken as the reference data. The bitmap compression is conducted for the data. The flow chart of the compression is shown in figure 3 .

\subsubsection{Threshold compression algorithm of high-frequency coefficient}

If the wavelet function and the orthonormal scaling function are adopted, the power quality signal can be decomposed into low-frequency coefficients and wavelet coefficients. The expression formula is shown as follows:

$E_{\text {signle }}=\sum_{k=-\infty}^{\infty}\left|d_{j}(\mathrm{k})\right|^{2}+\sum_{j=0}^{\infty} \sum_{k=-\infty}^{\infty}\left|d_{j}(\mathrm{k})\right|^{2}$

In this formula, the power quality with wavelet coefficients can be expressed as $E_{j}=\sum^{\infty}\left|d_{j}(\mathrm{k})\right|^{2}$ and the decomposition scale is represented $b=-\infty$.

This paper puts forward a data compression model of the high-frequency self-adaptive power quality threshold in order to reduce the complexity when the distribution of high-frequency coefficient changes tremendously. Thresholds are determined in accordance with the percentage of the high-frequency part.

$$
\begin{aligned}
& r=\lg \left(\mathrm{M} \times 10^{12}\right) \times u \\
& L n=\operatorname{int}(0.01 \times \text { length } \times \mathrm{r})
\end{aligned}
$$

In the above formula, the final threshold is $L n$, the number of original non-zero data is length, the correc- 
ICETA 2015

tion factor is $u$, the percentage of power quality is $\mathrm{M}$, and the threshold coefficient is $r$.

The specific step of the power quality data compression algorithm of wavelet transformation is to convert the two-dimensional discrete wavelet into non-zero values of high-frequency coefficients, record the data and sequence at last so as to obtain coefficients. The number of coefficients after the conversion is relatively small. Add column mark bits to realize further compression. 

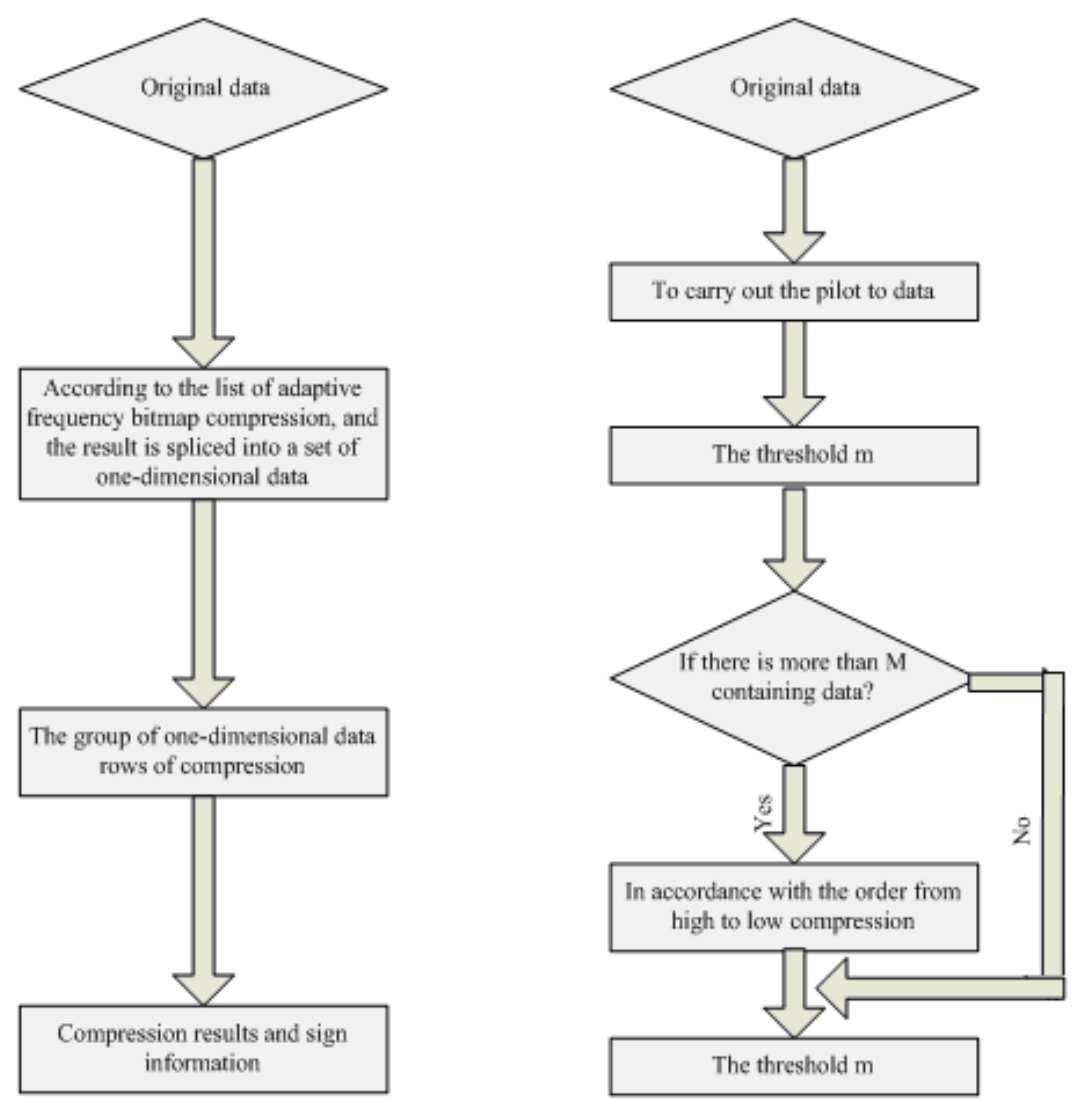

Figure 3. Flow chart of adaptive frequency bitmap compression algorithm

Table 1. Indicators of the one-dimensional wavelet algorithm

\begin{tabular}{llll}
\hline Signal type & $M S E / \%_{0}$ & $E R P / \%$ & $C R / \%$ \\
\hline Voltage flicker & 2.15 & 99.9994 & 11.37 \\
Short-term harmonic & 2.748 & 99.9991 & 5.95 \\
Voltage dip & 1.481 & 99.9997 & 11.44 \\
Voltage swell & 1.953 & 99.9995 & 10.90 \\
Voltage sag & 2.113 & 99.9997 & 11.06 \\
\hline
\end{tabular}

Table 2. Indicators the two-dimensional wavelet algorithm

\begin{tabular}{lllll}
\hline Signal type & $M S E / \%_{0}$ & $E R P / \%$ & $C R / \%$ & $S N R / \mathrm{dB}$ \\
\hline Voltage flicker & 2.2 & 99.995 & 2.26 & 53.782 \\
Short-term harmonic & 1.8 & 100.000 & 0.32 & 55.573 \\
Voltage dip & 2.4 & 100.000 & 2.48 & 50.916 \\
Voltage swell & 2.6 & 100.000 & 2.95 & 52.187 \\
Voltage sag & 2.5 & 100.000 & 2.87 & 51.936 \\
Normal signal & 1.6 & 100.000 & 1.23 & 55.606 \\
\hline
\end{tabular}

\section{LET TRANSFORMATION}

3 A SIMULATED ANALYSIS ON THE POWER QUALITY DATA COMPRESSION OF WAVE-
Statistics on the one-dimensional wavelet disturbance test results of five kinds of power qualities, namely 
voltage flicker, short-term harmonic, voltage dip, voltage swell and voltage sag that are provided in this paper. Software such as Matlab is used for the simulation and the normal voltage signals are taken as the reference. Results are provided in Table 1.

It can be known from the Table 1 that the data compression algorithm of the one-dimensional wavelet transformation is more ideal than the traditional algorithm. But it is relatively difficult to achieve the proportion which is less than 5\%.

In order to increase the compression ratio, the two-dimensional discrete wavelet is adopted in the simulation. Results of performance indicators are provided in the table which is shown in Table 2.

It can be concluded from the Table 2 that the two-dimensional algorithm is reduced by $9 \%$ compared with that in Table 1. It suggests that the data compression ratio is improved by the algorithm and the reconstruction error which is maintained is relatively small, so that the compression algorithm of power quality data is further improved.

\section{CONCLUSIONS}

1) By elaborating the wavelet transformation theory, this paper analyzes the development process of wavelet transformation, studies the property of wavelet from being continuous to being discrete, discusses the threshold coefficient compression algorithm after wavelet transformation, and lays a foundation for the study on power quality data compression.

2) Coefficients obtained from wavelet transformation can acquire reconstruction signals of high quality through the low-frequency and the high-frequency self-adaptive power quality threshold compression and data properties. Besides, the power quality data retention reaches up to $99 \%$ and the compression ratio is $3 \%$. Thus, it proves that the algorithm is suitable for the power quality data compression and signals can always keep low distortion. Therefore, the algorithm is excellent.

\section{ACKNOWLEDGEMENT}

The Project No.CDJZR12150026 is supported by the Fundamental Research Funds for the Central Universities.

\section{REFERENCES}

[1] Sun, P.X., Guo, Q., Wang, Q., Shi, W., Wang, Y. \& Wang, X.W. 2014. The power quality data compression algorithm of two-dimensional discrete wavelet transformation, Computer Application, 6(s1): 234-237.

[2] Tian, X. 2013. A Study on the Sampling Method of Multi-scale Power Quality Signal Compression Based on
Wavelet Transformation, Changsha: Central South University, 5: 5-8.

[3] Li, L. 2010. A Study on the Streaming Data Compression Algorithm Based on Wavelet Transformation, Changsha: Hunan University, 3: 6-9.

[4] Chen, H.F., Qiao, L. \& Liu, S.L. 2013. Disturbance recognition of computer quality based on wavelet transformation and SVM, Electrical Technology, (2): 12-17.

[5] S. Cui, Y. Wang \& J. E. Fowler, 2003. Multi-hypothesis motion compensation in the redundant wavelet domain. Int. Conf. on Image Processing, Barcelona, Spain, 2: 52-55.

[6] Wang, X.W., Wang, L. \& Miao, G.J., et al. 2012. The sampling and reconstruction method of transient-state and short-term of power quality disturbance signal compression, Power System Technology, 36(3): 190-195.

[7] QccPack-Quaution. 2003. Compression and Coding Library, pp: 121-124.

[8] Yang, X.F. 2010. A Study on the Network-based Power Quality Monitoring System Communication, Harbin: Harbin Institute of Technology.

[9] Zhang, D.F., et al. 2012. MATLAB Wavelet Analysis (2nd Edition), Beijing: China Machine Press. 